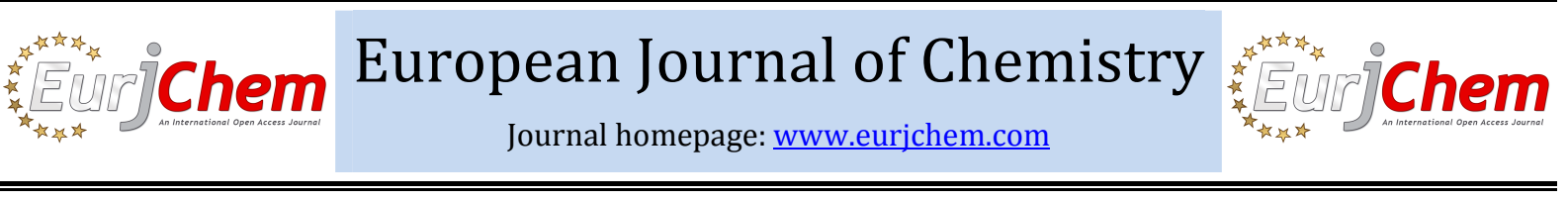

\section{Utility of (2,4-dioxo-1,4-dihydro-2H-quinazolin-3-yl)-acetic acid hydrazide in the synthesis of some heterocyclic nitrogen compounds}

\author{
Mamdouh Adly Hassan a, Maghrabi Ali Seleem b , Ahmed Mohamed Mosallem Younes b, \\ Mohamed Mobark Taha ${ }^{\mathrm{b}}$ and Abou-Bakr Haredi Abdel-Monsef $\mathrm{b}, *$ \\ a Pharmaceutical Chemistry Department, Faculty of Pharmacy, Sinai University, 45518, Arish, Egypt \\ b Chemistry Department, Faculty of Science, South Valley University, Qena, 83523, Egypt \\ ${ }^{*}$ Corresponding author at: Chemistry Department, Faculty of Science, South Valley University, Qena, 83523, Egypt. \\ Tel.: +20.96.5211281; fax: +20.96.5211279. E-mail address: bakooos2004@yahoo.com (A.H. Abdel-Monsef).
}

\section{ARTICLE INFORMATION}

Received: 01 March 2013

Received in revised form: 30 March 2013

Accepted: 30 March 2013

Online: 30 June 2013

\section{KEYWORDS}

\section{Isatine}

Acetic acid

Phthalimide

Phenylisothiocyanate

Quinazoline-2,4-dione

Tetrachlorophthalic anhydride

\section{Introduction}

Quinazolines are made up of two fused six member simple aromatic rings which represents one of the most important class of heterocycles possessing wide spectrum of biological activities and it considered the building block of many biologically active compounds that possess antibacterial [1-8], anti-inflammatory [1,2,7] and antifungal $[5,9,10]$. Quinazoline and pyrimidine derivatives are incorporated in a wide variety of pharmaceuticals. In addition, quinazoline and pyrimidine derivatives are attracting important applications in the field of medicinal chemistry; the pyrimidine ring is present in a large number of biological important compounds [11]. This encouraged us to synthesize new quinazoline derivatives attached to interesting heterocycles such as triazole, oxadiazole, pyrazole and phthalazine rings.

\section{Experimental}

\subsection{Instrumentation}

Melting points were uncorrected and determined on an electric melting point apparatus (Kofler). The IR spectra (KBr) were recorded on a Shimadzu 408 spectrometer. The ${ }^{1} \mathrm{H}$ NMR spectra were recorded using $300 \mathrm{MHz}$ Varian EM 390 spectrometer; chemical shifts are reported in ppm with TMS as an internal standard and are given in $\delta$ units. Electron impact mass spectra were obtained at $70 \mathrm{eV}$ with Shimadzu GC-MS (QP-2010 plus). ${ }^{13} \mathrm{C}$ NMR spectra were measured on a JEOL ECX instrument $400 \mathrm{MHz}$ in DMSO- $d_{6}$ and carried out at Jacobs University Bremen, Germany. Elemental analyses were carried out at the Microanalysis Unit at Cairo University. The purity of the compounds was detected by TLC.

\subsection{Synthesis}

\subsubsection{1-[(2,4-Dioxo-1,4-dihydro-2H-quinazolin-3-yl)- methylcarbonyl]-4-phenylthiosemicarbazide (4)}

To a mixture of hydrazide 3 (Scheme 1) $(2 \mathrm{~g}, 0.008 \mathrm{~mol})$ in absolute ethanol $(20 \mathrm{~mL})$ phenyl isothiocyanate $(0.011 \mathrm{~mol})$ was added and the reaction mixture was heated under reflux for $4 \mathrm{hrs}$., then left to cool. The solid that separated was collected by filtration crystallized from ethanol to afford compound 4 (1.7 g, $0.004 \mathrm{~mol}$ ) as white crystals (Scheme 2). Yield: 1.7 g, 54 \%. M.p.: 220 'C. FT-IR (KBr, v, cm$\left.{ }^{-1}\right): 3339,3304$ 3292, 3265 (NH's), 1733, 1661, 1635 (C=0's). ${ }^{1} \mathrm{H}$ NMR (300 MHz, DMSO- $\left.d_{6}, \delta, \mathrm{ppm}\right): 4.7\left(\mathrm{~s}, 2 \mathrm{H}, \mathrm{CH}_{2}\right), 7.1-7.9$ (m, 9H, arom.), $9.3(\mathrm{~s}, 1 \mathrm{H}, \mathrm{NH}), 9.7(\mathrm{~s}, 1 \mathrm{H}, \mathrm{NH}), 10.4(\mathrm{~s}, 1 \mathrm{H}, \mathrm{NH}), 11.6(\mathrm{~s}, 1 \mathrm{H}$, $\mathrm{NH}) .{ }^{13} \mathrm{C}$ NMR $\left(100 \mathrm{MHz}, \mathrm{DMSO}-d_{6}, \delta, \mathrm{ppm}\right): 41.7,113.6,115.2$, $120.8,122.7,124.4,125.9,127.3,128.1,135.2,138.9,139.3$, 150.2, 162, 167. MS (m/z, \%): 369 (3.0 \%). Anal. calcd. for $\mathrm{C}_{17} \mathrm{H}_{15} \mathrm{~N}_{5} \mathrm{O}_{3} \mathrm{~S}: \mathrm{C}, 55.28 ; \mathrm{H}, 4.08$; N,18.96. Found: C, 55.48; H, 4.2; N, $19.02 \%$.

2.2.2. 3-(5-Mercapto-4-phenyl-4H-[1,2,4]triazol-3-ylmethyl)1H-quinazoline-2,4-dione (5)

Compound 4 was refluxed in $\mathrm{NaOH}$ solution $(10 \mathrm{~mL})$ for 6 hrs. The mixture was left to cool, filtered, and the filtrate was acidified with dil. $\mathrm{HCl}$. The solid obtained was filtered off, and crystallized from benzene/ethanol to give compound $\mathbf{5}$ as white crystals (Scheme 2). M.p.: $>300{ }^{\circ} \mathrm{C}$. FT-IR $\left(\mathrm{KBr}, v, \mathrm{~cm}^{-1}\right)$ : $3126(\mathrm{NH}), 2510(\mathrm{SH}), 1721,1663$ (C=0's). ${ }^{1} \mathrm{H}$ NMR (300 MHz, DMSO- $\left.d_{6}, \delta, \mathrm{ppm}\right): 4.9\left(\mathrm{~s}, 2 \mathrm{H}, \mathrm{CH}_{2}\right), 7.1-7.9(\mathrm{~m}, 9 \mathrm{H}$, arom.), 11.6 (s, $1 \mathrm{H}, \mathrm{NH}), 13.8(\mathrm{~s}, 1 \mathrm{H}, \mathrm{SH})$.

European Journal of Chemistry 

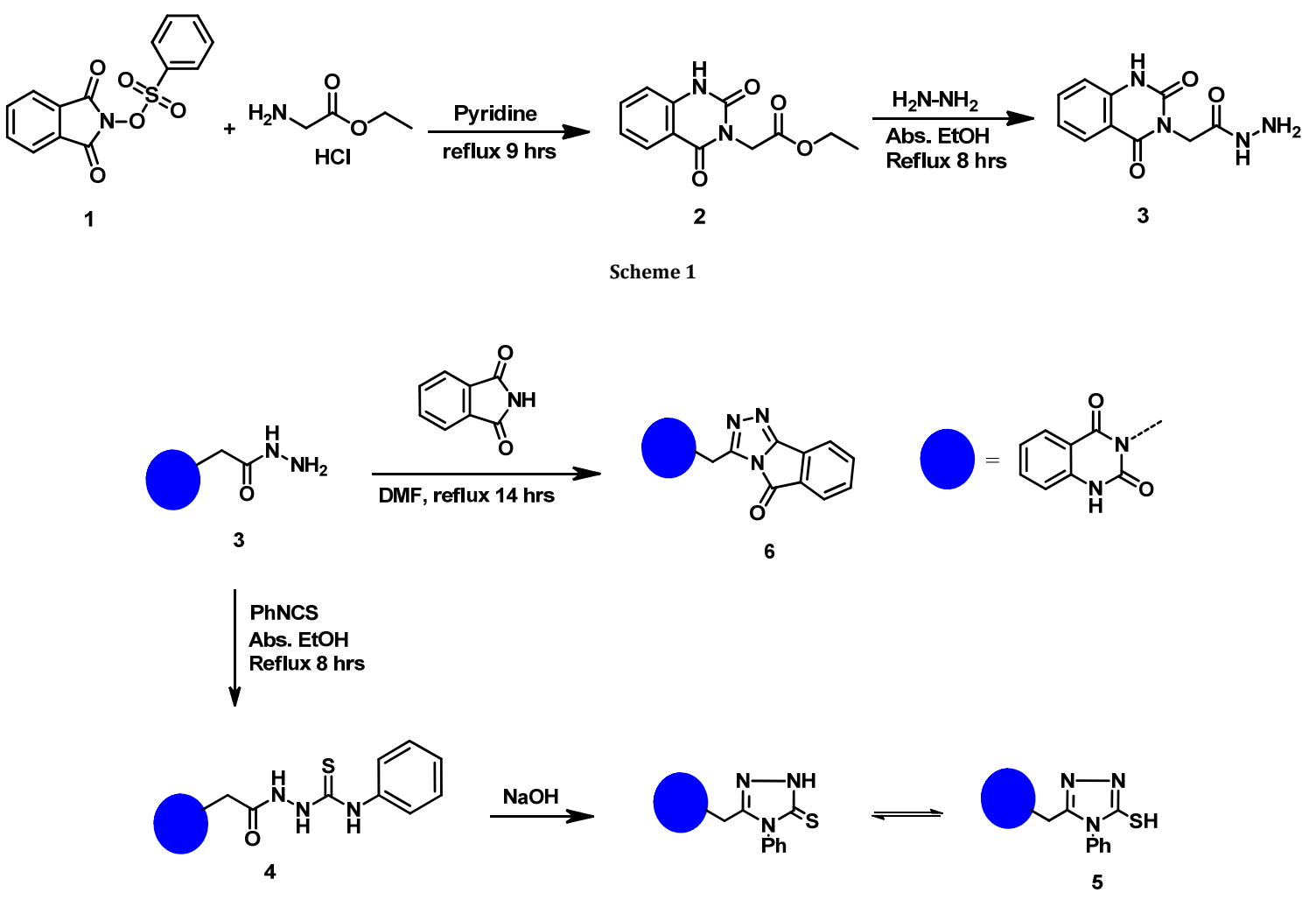

Scheme 2

${ }^{13} \mathrm{C}$ NMR (100 MHz, DMSO- $\left.d_{6}, \delta, \mathrm{ppm}\right): 35.5,113.2,115.2,120.8$, $122.7,127.3,127.9,129.4,133,135.3,139.2,148.2,148.5$, 161.3, $167.9 \%$ ). Anal. calcd. for $\mathrm{C}_{17} \mathrm{H}_{13} \mathrm{~N}_{5} \mathrm{O}_{2} \mathrm{~S}$ : C,58.11; $\mathrm{H}, 3.73$; N,19.93. Found: C, 58.31; H, 3.75; N, 20.8\%.

\subsubsection{3-((5-oxo-5H-[1,2,4]triazolo[3,4-a]isoindol-3-yl) methyl)quinazoline-2,4(1H,3H)-dione (6)}

Heating of the hydrazide 3 (1 g, $0.004 \mathrm{~mol})$ with phthalimide $(0.75 \mathrm{~g}, 0.005 \mathrm{~mol})$ in DMF $(20 \mathrm{~mL})$ under reflux for 14 hours gave after cooling a solid product which was filtered off and crystallized from benzene to give compound $\mathbf{6}$ as yellow crystals (Scheme 2). Yield: $0.4 \mathrm{~g}$, 59 \%. M.p.: $224{ }^{\circ} \mathrm{C}$. FT-IR (KBr, v, cm-1): 3200 (NH), 1774, 1752, 1603 (C=0's). MS ( $m / z, \%): 345$ (1.46 \%). Anal. calcd. for $\mathrm{C}_{18} \mathrm{H}_{11} \mathrm{~N}_{5} \mathrm{O}_{3}: \mathrm{C}, 62.62 ; \mathrm{H}$, 3.21; N, 20.28. Found: C, 62.9; H, 3.24; N, 20.51\%.

2.2.4. 3-Amino-1-[2-(2,4-dioxo-1,4-dihydro-2H-quinazolin-3yl)-acetyl]-5-phenyl-1H-pyrazole-4-carbonitrile (7)

Benzylidine malononitrile $(0.27 \mathrm{~g}, 0.002 \mathrm{~mol})$ in ethanol $(20 \mathrm{~mL})$ and piperidine as a catalyst was added to hydrazide 3 $(0.4 \mathrm{~g}, 0.002 \mathrm{~mol})$ and the reaction mixture was refluxed for 9 hrs., the formed solid product was filtered off and recrystallized from ethanol to afford compound $7(0.16 \mathrm{~g}, 0.0004 \mathrm{~mol})$ as white crystal (Scheme 3). Yield: 0.16 g, $66 \%$. M.p.: $>300{ }^{\circ} \mathrm{C}$. FTIR (KBr, v, cm-1): $3300(\mathrm{NH}), 3198\left(v \mathrm{NH}_{2}\right), 2250(\mathrm{CN}), 1741$, 1637 (C=O's). ${ }^{1} \mathrm{H}$ NMR (300 MHz, DMSO- $\left.d_{6}, \delta, \mathrm{ppm}\right): 4.9$ (s, $2 \mathrm{H}$, $\mathrm{CH}_{2}$ ), 7.2-7.92 (m, 9H, arom.), 11.5 (s, 2H, $\left.\mathrm{NH}_{2}\right), 11.7$ (s, $1 \mathrm{H}$, $\mathrm{NH}) .{ }^{13} \mathrm{C}$ NMR (100 MHz, DMSO- $\left.d_{6}, \delta, \mathrm{ppm}\right): 41.4,113.4,115.2$, $118.8,119.3,122.6,126.8,127,127.3,128.7,129.9,133.9$, 135.1, 139.2, 144, 150, 161.7, 168. Anal. calcd. for $\mathrm{C}_{20} \mathrm{H}_{14} \mathrm{~N}_{6} \mathrm{O}_{3}$ : C,62.17; H,3.65; N,21.75. Found: C, 62.38; H, 3.67; N, 22.02\%.
2.2.5. (2,4-Dioxo-1,4-dihydro-2H-quinazolin-3-yl)-acetic acid (2-oxo-1,2-dihydro-indol-3-ylidene)-hydrazide (8)

Treatment of hydrazide $3(0.4 \mathrm{~g}, 0.002 \mathrm{~mol})$ with isatine $(0.25 \mathrm{~g}, 0.002 \mathrm{~mol})$ in acetic acid $(20 \mathrm{~mL})$ and reflux for $9 \mathrm{hrs}$. afforded compound $8(0.3 \mathrm{~g}, 0.001 \mathrm{~mol})$ as yellow crystals (Scheme 3). Yield: $0.3 \mathrm{~g}, 50 \%$ \%. M.p.: $298^{\circ} \mathrm{C}$. FT-IR $\left(\mathrm{KBr}, v, \mathrm{~cm}^{-1}\right)$ : 3306, 3222, 3165 (NH's), 1733, 1752, 1635 (C=0's). ${ }^{1} \mathrm{H}$ NMR (300 MHz, DMSO- $\left.d_{6}, \delta, \mathrm{ppm}\right): 5.1\left(\mathrm{~s}, 2 \mathrm{H}, \mathrm{CH}_{2}\right), 6.9-7.9(\mathrm{~m}, 8 \mathrm{H}$, arom.), $11.2(\mathrm{~s}, 1 \mathrm{H}, \mathrm{NH}), 11.67(\mathrm{~s}, 1 \mathrm{H}, \mathrm{NH}), 12.6(\mathrm{~s}, 1 \mathrm{H}, \mathrm{NH}) .{ }^{13} \mathrm{C}$ NMR (100 MHz, DMSO- $\left.d_{6}, \delta, \mathrm{ppm}\right): 40.3,111.1,113.4,115.3$, $119.4,120.8,122.5,122.8,127.4,131.7,135.3,139.3,142.5$ 149.9, 161.7, 162.4, 199.6, 205.3. MS ( $\mathrm{m} / \mathrm{z}, \%): 363$ (6.0 \%). Anal. calcd. for $\mathrm{C}_{18} \mathrm{H}_{13} \mathrm{~N}_{5} \mathrm{O}_{4}$ : C,59.50; $\mathrm{H}, 3.61 ; \mathrm{N}, 19.28$. Found: C, 59.71; H, 3.63; N, 20.32\%.

2.2.6. 3-[2-Oxo-2-(5,6,7,8-tetrachloro-1,4-dioxo-3,4-dihydro1H-phthalazin-2-yl)-ethyl]-1H-quinazoline-2,4-dione (9)

Tetrachlorophthalic anhydride $(1.2 \mathrm{~g}, 0.004 \mathrm{~mol})$ in acetic acid $(20 \mathrm{~mL})$ was added to hydrazide $3(1 \mathrm{~g}, 0.004 \mathrm{~mol})$, then the reaction mixture was refluxed for 9 hrs., the formed solid product was filtered off and recrystallized from dioxane to give compound 9 as white crystals (Scheme 3). Yield: $1.2 \mathrm{~g}, 57 \%$. M.p.: $>310{ }^{\circ} \mathrm{C}$. FT-IR $\left(\mathrm{KBr}, v, \mathrm{~cm}^{-1}\right): 3304(\mathrm{NH}), 1661,1635$ (C=0's). ${ }^{1} \mathrm{H}$ NMR (300 MHz, DMSO- $\left.d_{6}, \delta, \mathrm{ppm}\right): 4.7\left(\mathrm{~s}, 2 \mathrm{H}, \mathrm{CH}_{2}\right)$, 7.0-7.9 (m, 4H, arom.), 11.2 (s, 1H, NH), 11.6 (s, 1H, NH). MS (m/z, \%): 502 (1.27\%). Anal. calcd. for $\mathrm{C}_{18} \mathrm{H}_{8} \mathrm{Cl}_{4} \mathrm{~N}_{4} \mathrm{O}_{5}$ : C,43.06; H,1.61; N,11.16. Found: C, 43.28; H, 1.63; N, 11.92\%. 
<smiles>Cn1c(=O)[nH]c2ccccc2c1=O</smiles><smiles>O=C(CC1CCCCC1)n1[nH]c(=O)c2c(Cl)c(Cl)c(Cl)c(Cl)c2c1=O</smiles>

9<smiles>CC(=O)NNC(=O)CC1CCCCC1</smiles>

10

AcOH, reflux

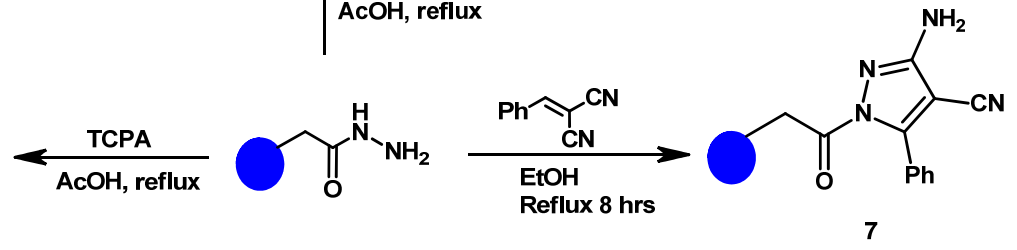

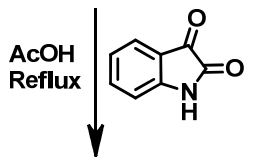<smiles>O=C(Cc1ccccc1)N/N=C1\C(=O)Nc2ccccc21</smiles>

8

Scheme 3<smiles>CCOC(=O)Cn1c(=O)[nH]c2ccccc2c1=O</smiles>

Scheme 4

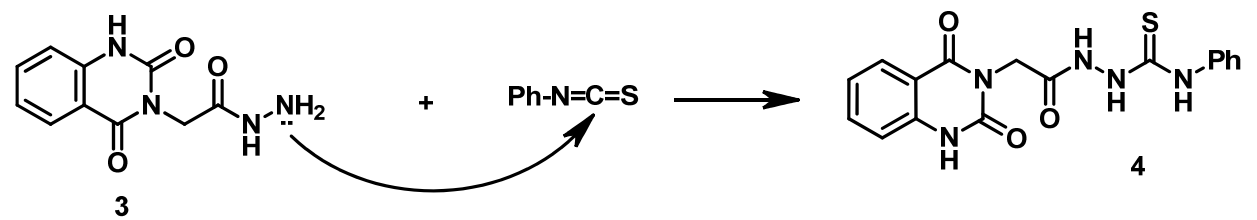

Scheme 5

\subsubsection{Acetic acid $N^{\prime}-[2-(2,4-d i o x o-1,4-d i h y d r o-2 H-$ quinazolin-3-yl)-acetyl]-hydrazide (10)}

Refluxing of hydrazide $3(0.5 \mathrm{~g}, 0.002 \mathrm{~mol})$ with acetic acid $(20 \mathrm{~mL})$ for $8 \mathrm{hrs}$. On cooling, the separated solid was filtered off and crystallized from acetic acid to give compound $\mathbf{1 0}(0.4 \mathrm{~g}$, $0.0015 \mathrm{~mol}$ ) as white crystals (Scheme 3). Yield: $0.4 \mathrm{~g}, 66 \%$. M.p.: $>300{ }^{\circ} \mathrm{C}$. FT-IR $\left(\mathrm{KBr}, v, \mathrm{~cm}^{-1}\right): 3304,3282,3265$ (NH's), 1733, 1732, 1635 (C=0's). ${ }^{1} \mathrm{H}$ NMR (300 MHz, DMSO- $d_{6}, \delta$, ppm): 1.9 (s, 3H, $\mathrm{CH}_{3}$ ), 4.5 (s, $2 \mathrm{H}, \mathrm{CH}_{2}$ ), 7.1-7.9 (m, $4 \mathrm{H}$, arom.), $9.8(\mathrm{~s}, 1 \mathrm{H}, \mathrm{NH}), 10.1(\mathrm{~s}, 1 \mathrm{H}, \mathrm{NH}), 11.5(\mathrm{~s}, 1 \mathrm{H}, \mathrm{NH}) .{ }^{13} \mathrm{C}$ NMR $(100$ MHz, DMSO- $\left.d_{6}, \delta, \mathrm{ppm}\right): 20.9,41.6,114.1,115.7,123.1,127.9$, 135.7, 139.9, 150.4, 162.3, 166.3, 168.4. MS ( $\mathrm{m} / \mathrm{z}, \%): 276$ (3.0 \%). Anal. calcd. for $\mathrm{C}_{12} \mathrm{H}_{12} \mathrm{~N}_{4} \mathrm{O}_{4}$ : C,52.18; $\mathrm{H}, 4.37 ; \mathrm{N}, 20.28$. Found: C, 52.39; H, 4.39; N, $20.35 \%$.

\section{Results and discussion}

In this work, we were able to introduce the amino group which is present in glycine ethyl ester hydrochloride into the sulphonyloxy system in $N$-phenylsulphonyloxyphthalimide $\mathbf{1}$ to obtain (2,4-dioxo-1,4-dihydro-2H-quinazolin-3-yl)-acetic acid ethyl ester 2 which on treatment with hydrazine hydrates in ethanol gave the hydrazide 3 (Scheme 1) [12]. Scheme 4 outlines the synthetic pathway used to obtain $(2,4$-dioxo-1,4dihydro- $2 H$-quinazolin-3-yl)-acetic acid hydrazide 3 [12]. The route outlined in Scheme 2 shown that hydrazide 3 used as a starting material for synthesis of some new quinazoline derivatives attached to interesting heterocycles such as triazole, oxadiazole, pyrazole and phthalazine rings which have biological effects $[13,14]$, and many applications were they are used as fungicidal and bacterial reagents [15]. 

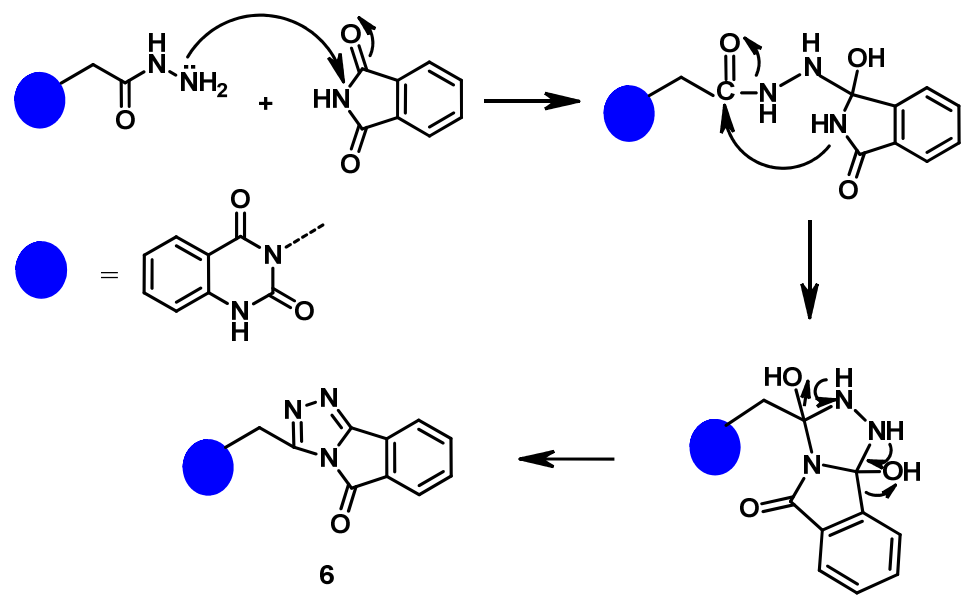

Scheme 6

Scheme 5 and 6 outline the synthetic pathway used to synthesis 1-[(2,4-dioxo-1,4-dihydro-2H-quinazolin-3-yl)-methylcarbonyl]-4-phenylthiosemicarbazide 4 and 3-((5-oxo- $5 \mathrm{H}$ $[1,2,4]$ triazolo[3,4-a]isoindol-3-yl)methyl)quinazoline-2,4(1H, $3 \mathrm{H}$ )-dione (6). Scheme 3 shows the results of treatment of hydrazide 3 with benzylidine malononitrile, isatine, tetrachloro phthalic anhydride and acetic acid. Treatment of hydrazide $\mathbf{3}$ with benzylidine malononitrile in ethanol under reflux gave 3amino-1-[2-(2,4-dioxo-1,4-dihydro- $2 H$-quinazolin-3-yl)-acetyl]-5-phenyl-1H-pyrazole-4-carbonitrile, 7 , which contains pyrazole ring. As a part of our program aimed to synthesize new rings attached to quinazoline ring as we notice in compounds 8 and 9 which contain indol-2-one and phthalazine rings, respectively, by reacting of hydrazide 3 with isatine and tetrachlorophthalic anhydride, respectively. Treatment of hydrazide 3 with acetic acid under reflux gave acetic acid $N^{\prime}$-[2(2,4-dioxo-1,4-dihydro-2H-quinazolin-3-yl)-acetyl]-hydrazide,

\section{0.}

\section{Conclusion}

The synthesis of new quinazolindione derivatives was achieved and structural verification was achieved by spectral and physical analysis.

\section{Acknowledgments}

The authors are grateful to the spirit of Prof. Dr. Youssef Hassan Ebeed, Faculty of Science, South Valley University, Rami Al-Oweini and Ibrahim Mohamed Ismael for their help in analysis of some samples.

\section{References}

[1]. Bakhite, E. A.; Radwan, S. M.; El-deen, A. M. K. J. Chin. Chem. Soc. 2000, 47(5), 1105-1114.

[2]. Younes, M. I.; Abbas, H. H.; Metwally, S. A. M. Pharmazine 1999, 46(2), 98-100.

[3]. Rideout, J. L.; Krenitesky, T. A.; Chao, E. Y.; Elion, G. B.; Williams, R. B.; Latter, V. S. J. Med. Chem. 1983, 26(10), 1489-1494.

[4]. Rideout, J. L.; Krenitesky, T. A.; Koszalka. G. W.; Coln, N. K.; Chao, E. Y.; Elion, G. B.; Latter, V. S.; Williams, R. B. J. M. Chem. 1982, 25(9), 10401044.

[5]. Marie, M. G.; Aly, D. M.; Mishrikey, M. M. Bull. Chem. Soc. Jpn. 1992, 65(12), 3419-3422.

[6]. Krenitesky, T. A.; Rideout, J. L.; Koszalka, G. W.; Inmmon, R. B.; Chao, E. Y.; Elion, G. B. J. Med. Chem. 1982, 25(1), 32-35.

[7]. Gatta, F.; Perotti, F.; Gradoni, L.; Gramiccia, M.; Orsini, S.; Palazzo, G.; Rossi, V. Eur. J. Med. Chem. 1990, 30, 419-424.

[8]. Ugarkar, B. G.; Cottam, H. B.; Mekernan, P. A.; Robins, R. K.; Revankar, G. R. J. Med. Chem. 1984, 27(8), 1026-1030
[9]. Makara, G. M.; Ewing, W.; winter, E. J. Org. Chem. 2001, 66(17), 57835787.

[10]. Mishara, B.; Muddin, N. Indian J. Chem. 1989, 28B, 337-346.

[11]. Ostrowski, S.; Swat, J.; Makosza, M. Arkivoc 2000, 1(6), 905-908.

[12]. Hassan, M. A.; Seleem, M. A.; Younes, A. M. M.; Taha, M. M.; AbdelMonsef, A. H. Eur.J Chem. 2013, 4(2), 121-123.

[13]. Ram, V.; Pandey, H. N. Chem. Pharm. Bull. (Jpn.) 1974, 22, 2778-2783.

[14]. Parmar, S.; Chaudhary, M.; Chaudhary, S.; Kumar, S.; Spiro, H. J. Pharm. Sci. 1977, 66, 971-975.

[15]. Jain, R.; Shukla, A. Indian J. Chem. Soc. 1990, 67, 575-579. 